\title{
Correlation and prognostic value of osteopontin and Bcl-2 in hepatocellular carcinoma patients after curative resection
}

\author{
BIAO DENG ${ }^{*}$, XIAO-FEI ZHANG* ${ }^{*}$ XU-CHAO ZHU, HUA HUANG, HU-LIANG JIA, \\ QING-HAI YE, QIONG-ZHU DONG and LUN-XIU QIN
}

\begin{abstract}
Liver Cancer Institute and Zhongshan Hospital, Institutes of Biomedical Sciences, Fudan University, Key Laboratory of Carcinogenesis and Cancer Invasion, Ministry of Education, Shanghai 200032, P.R. China
\end{abstract}

Received July 4, 2013; Accepted August 9, 2013

DOI: $10.3892 /$ or.2013.2737

\begin{abstract}
Osteopontin (OPN) may facilitate tumorigenesis and metastasis through prevention of tumor cells from apoptosis. Although previous studies have suggested involvement of enhanced $\mathrm{Bcl}-2$ protein family expression, the role of OPN together with Bcl-2 in hepatocellular carcinoma (HCC) remains unknown. In this study, we used western blotting to detect the OPN and Bcl-2 expression levels in cell lines with different OPN backgrounds and HCC tissues, and tumor tissue microarrays to examine OPN and Bcl-2 expression levels in $454 \mathrm{HCC}$ cases. The Kaplan-Meier method and log-rank test were applied to investigate the predictive values of OPN and Bcl-2 in HCC patients. In vitro assays indicated that OPN expression increased concordantly with increasing metastatic potential in MHCC97-H, MHCC97-L, HepG2 and SMMC-7721 cell lines by western blotting, whereas Bcl-2 expression declined. In addition, Bcl-2 was highly upregulated in OPN knockdown MHCC97-H cell lines. Furthermore, in HCC tissues, it was confirmed that OPN levels were also significantly higher in recurrent tumor tissues compared to non-recurrent tissues by western blotting $(\mathrm{p}<0.001)$, whereas the contrary occurred in Bcl-2 ( $\mathrm{p}=0.046)$. Using immunohistochemistry analysis, patients with higher OPN levels had significantly shorter median survival time and recurrence time compared to the lower ones, although the opposite occurred in Bcl-2 levels. Of note, when OPN and Bcl-2 were combined, we found that the co-index of OPN/Bcl-2 was an independent prognostic factor for both overall survival $(\mathrm{p}<0.001)$ and time to recurrence
\end{abstract}

Correspondence to: Professor Lun-Xiu Qin or Dr Qiong-Zhu Dong, Liver Cancer Institute and Zhongshan Hospital,Institutes of Biomedical Sciences, Fudan University, 180 Feng Lin Road, Shanghai 200032, P.R. China

E-mail: qin.lunxiu@zs-hospital.sh.cn

E-mail: qzhdong@yahoo.com.cn

*Contributed equally

Key words: hepatocellular carcinoma, osteopontin, Bcl-2, recurrence, prognosis $(\mathrm{p}<0.001)$. Our findings demonstrate that OPN/Bcl-2 expression is a promising independent predictor of recurrence and survival in HCC. Additionally, Bcl-2 levels may be regulated by OPN in the HCC microenvironment.

\section{Introduction}

Osteopontin (OPN), a secreted non-collagenous, sialic-acidrich, chemokine-like extracellular matrix (ECM) protein, has been implicated as an important mediator and a potential therapeutic target of cancer metastasis (1). OPN could inhibit the apoptosis of adherent endothelial cells induced by growth factors and cytokine depletion by enhancing Bcl-xL expression (2). Bcl-2 has been demonstrated to play important roles in the development and progression of several types of cancer (3-5), and can be an independent predictor of cancer outcome $(6,7)$. Bcl-2 has also been found to play an important role in resistance to 5-fluorouracil-caused cell death of hepatocellular carcinoma (HCC) (8). In a previous report, OPN silencing reduced the expression of integrin $\alpha \mathrm{v}, \beta 1$ and $\beta 3$, blocked NF- $\kappa$ B activation, suppressed Bcl-2/Bcl-xL and XIAP expression, increased Bax expression, and induced a mitochondria-mediated apoptosis (9). However, the correlation between OPN and BCL-2 in HCC and their potential roles in prognosis remain unclear.

In the present study, we examined the expression levels of OPN and Bcl-2 in HCC cell lines and tumor tissues from 454 cases of HCC patients to investigate the correlation between $\mathrm{OPN}$ and $\mathrm{Bcl}-2$ and its prognostic values for $\mathrm{HCC}$ patients. We found that the combination of OPN and Bcl-2 could provide prognostic information for HCC patients. Bcl-2 levels may be regulated by OPN in the HCC microenvironment.

\section{Materials and methods}

Patients and cell lines. Four hundred and fifty-four patients undergoing curative resection for HCC at the Liver Cancer Institute, Zhongshan Hospital, Fudan University, between 2004 and 2006, were enrolled in this study. For each patient, the diagnosis of HCC was confirmed by pathologic examination and complete follow-up data were available. The clinicopathologic characteristics of patients in the tumor tissue microarray (TMA) are summarized in Table I. Follow-up was completed 
Table I. Associations between OPN/BCL-2 density as detected by immunohistochemical staining of tissue microarrays and clinicopathological characteristics of HCC patients $(n=454)$.

\begin{tabular}{|c|c|c|c|c|c|c|}
\hline \multirow[b]{2}{*}{ Variable } & \multicolumn{2}{|c|}{ Tumoral OPN density, $\mathrm{n}$} & \multirow[b]{2}{*}{ P-value } & \multicolumn{2}{|c|}{ Tumoral BCL-2 density, $\mathrm{n}$} & \multirow[b]{2}{*}{ P-value } \\
\hline & High $(n=227)$ & Low $(n=227)$ & & $\operatorname{High}(\mathrm{n}=227)$ & Low $(n=227)$ & \\
\hline \multicolumn{7}{|l|}{ Gender } \\
\hline Female & 35 & 42 & 0.381 & 39 & 38 & 0.901 \\
\hline Male & 192 & 185 & & 188 & 189 & \\
\hline \multicolumn{7}{|l|}{ Age, years } \\
\hline$\leq 51$ & 123 & 111 & 0.260 & 117 & 117 & 1.000 \\
\hline$>51$ & 104 & 116 & & 110 & 110 & \\
\hline \multicolumn{7}{|c|}{ Preoperative AFP (ng/ml) } \\
\hline$\leq 20$ & 86 & 79 & 0.495 & 78 & 87 & 0.380 \\
\hline$>20$ & 141 & 148 & & 149 & 140 & \\
\hline \multicolumn{7}{|l|}{ HBsAg } \\
\hline Negative & 26 & 10 & 0.006 & 19 & 17 & 0.728 \\
\hline Positive & 201 & 217 & & 208 & 210 & \\
\hline \multicolumn{7}{|c|}{ Liver cirrhosis } \\
\hline No & 22 & 25 & 0.664 & 27 & 20 & 0.281 \\
\hline Yes & 205 & 202 & & 200 & 207 & \\
\hline \multicolumn{7}{|c|}{ ALT (units/l) } \\
\hline$\leq 75$ & 200 & 200 & 1.000 & 200 & 200 & 1.000 \\
\hline$>75$ & 27 & 27 & & 27 & 27 & \\
\hline \multicolumn{7}{|c|}{ Tumor size $(\mathrm{cm})$} \\
\hline$\leq 5$ & 157 & 174 & 0.073 & 158 & 173 & 0.113 \\
\hline$>5$ & 70 & 53 & & 69 & 54 & \\
\hline \multicolumn{7}{|c|}{ Tumor encapsulation } \\
\hline Complete & 122 & 114 & 0.452 & 108 & 128 & 0.060 \\
\hline None & 105 & 113 & & 119 & 99 & \\
\hline \multicolumn{7}{|c|}{ Vascular invasion } \\
\hline No & 161 & 145 & 0.109 & 152 & 154 & 0.841 \\
\hline Yes & 66 & 82 & & 75 & 73 & \\
\hline \multicolumn{7}{|c|}{ BCLC stage } \\
\hline A & 151 & 143 & 0.432 & 147 & 147 & 1.000 \\
\hline $\mathrm{B} / \mathrm{C}$ & 76 & 84 & & 80 & 80 & \\
\hline \multicolumn{7}{|c|}{ Tumor differentiation } \\
\hline I-II & 190 & 156 & $<0.001$ & 169 & 177 & 0.378 \\
\hline III-IV & 37 & 71 & & 58 & 50 & \\
\hline
\end{tabular}

ALT, alanine aminotransferase; BCLC, Barcelona Clinic Liver Cancer staging system; HBsAg, hepatitis B surface antigen; NS, not significant $\left(\chi^{2}\right.$ tests). $\chi^{2}$ tests were conducted for all analyses. OPN, osteopontin; HCC, hepatocellular carcinoma. AFP, $\alpha$-fetoprotein.

in March 2012. The mean survival was $61.6 \pm 1.9$ months. Follow-up after surgery included serum $\alpha$-fetoprotein (AFP), ultrasonography and chest X-ray every 2-3 months according to the postoperative time $(8,10)$. The diagnosis of recurrence was based on typical imaging appearance in computed tomography and/or magnetic resonance imaging scan and an elevated AFP level. Overall survival (OS) was defined as the interval between the date of surgery and mortality. Time to recurrence (TTR) was defined as the time between the start of surgery and the first report of recurrence (excluding patients who succumbed to non-liver cancer causes before recurrence) $(11,12)$. For patients who had not experienced a recurrence at the time of mortality or last follow-up, TTR was censored at the date of mortality or the last follow-up.

The study was approved by the Research Ethics Committee of Zhongshan Hospital, Fudan University (Shanghai, China). Snap-frozen or paraffin-embedded tissue specimens were obtained from these patients after receiving written informed 

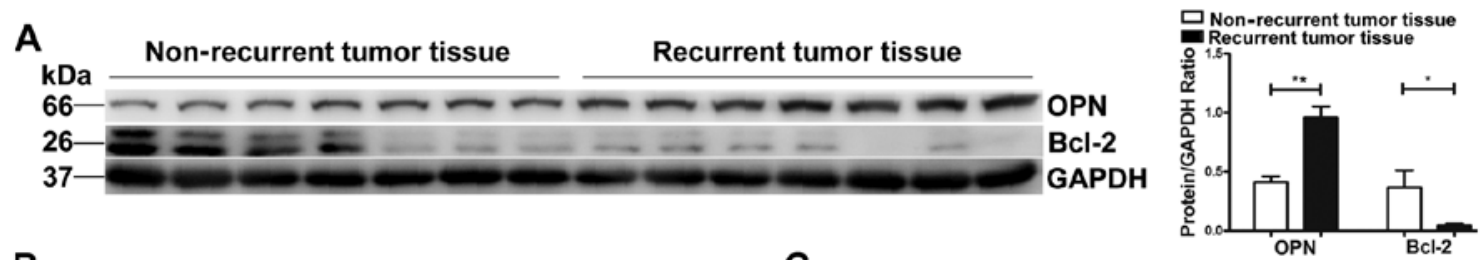

B

C
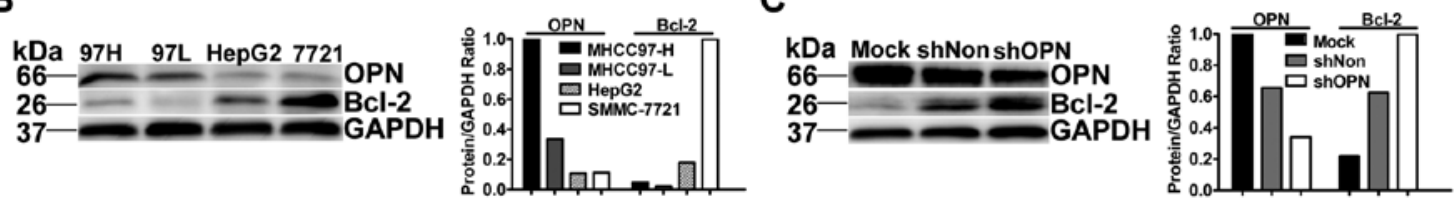

Figure 1. Protein expression levels of OPN and Bcl-2 in HCC tissues and cell lines by western blotting. (A) OPN expressed highly in recurrent tumor tissues but low in non-recurrent ones. The trend of Bcl-2 expression was the opposite. In the relative quantitative analysis below, OPN was significantly higher in recurrent than in non-recurrent tumor tissue $\left({ }^{* *} \mathrm{p}<0.001\right)$. Bcl-2 was significantly higher in non-recurrent than in recurrent tumor tissue $\left({ }^{*} \mathrm{p}=0.046\right)$. $(\mathrm{B}) \mathrm{OPN}$ and $\mathrm{Bcl}-2$ expression levels were detected by western blotting in MHCC97-H, MHCC97-L, HepG2 and SMMC-7721 cell lines. Relative quantization is also shown. OPN expression increased with increasing metastatic potential in human HCC cell lines. Bcl-2 decreased with increasing metastatic potential. (C) Expression levels of OPN and Bcl-2 in MHCC97-H cells transfected with mock, shNon and shOPN. Expression levels of OPN in MHCC97-H cells transfected with shOPN decreased significantly compared with mock and shNon. Bcl-2 expression increased significantly in shOPN MHCC97-H cells. A monoclonal antibody against GAPDH was used as an internal control. OPN, osteopontin; HCC, hepatocellular carcinoma.

consent. Fresh tissues for western blotting were collected immediately after resection, frozen in liquid nitrogen, and stored at $-80^{\circ} \mathrm{C}$.

MHCC97-H and MHCC97-L (human HCC cell lines with different metastatic potentials established by authors' institute from the same parent cell line), SMMC-7721, HepG2 cells (low-metastatic; American Type Culture Collection) were used in this study. Cells were cultured in Dulbecco's Modified Eagle's Medium (DMEM) (HyClone, Logan, UT, USA) supplemented with $10 \%$ (vol/vol) fetal bovine serum (FBS; Gibco-BRL, New York, NY, USA) at $37^{\circ} \mathrm{C}$ in a humidified incubator containing $5 \% \mathrm{CO}_{2}$.

Tissue microarray immunohistochemistry analysis for OPN and $\mathrm{Bcl}-2$. Tissue microarrays were constructed as previously described (13). Briefly, duplicate 1-mm-diameter cylinders were obtained from 2 different areas of the donor blocks and transferred to the recipient paraffin block at defined array positions (Shanghai Biochip Co., Ltd.). Consecutive 4- $\mu$ m-thick sections were obtained on 3-aminopropyltriethoxysilanecoated slides (14).

Immunohistochemistry was carried out using a two-step protocol according to the manufacturer's instructions. Briefly, the sections were dewaxed in xylene and graded alcohols, hydrated, and washed in PBS. After endogenous peroxidase was inhibited by $3 \% \mathrm{H}_{2} \mathrm{O}_{2}$ for $30 \mathrm{~min}$, the sections were pretreated in a microwave oven (14 min in sodium citrate buffer; $\mathrm{pH}=6$ ), nonspecific binding sites were blocked with Protein Block (Novocastra Laboratories, Newcastle upon Tyne, UK), and then the tissues were incubated with primary antibodies for $12 \mathrm{~h}$ in a moist chamber at $4^{\circ} \mathrm{C}$. Primary antibodies used for detecting the expression levels of OPN and Bcl-2 were mouse anti-human OPN antibody (1:100) and rabbit antihuman Bcl-2 antibody (1:100; both from Abcam, Cambridge, $\mathrm{UK})$. The components of the Envision-plus detection system were applied (EnVision+/HRP/Mo; Dako, Carpinteria, CA, USA). Reaction products were visualized by incubation with 3,3-diaminobenzidine solution. Negative controls were treated identically but with the primary antibodies omitted (12).
The images of 4 representative fields were captured by the Leica QWin Plus version 3 software and identical settings were used for each image under high-power magnification (x200). The density of positive staining was evaluated using a Leica CCD camera DFC420 connected to a Leica DMIRE2 microscope (Leica Microsystems Imaging Solutions, Cambridge, UK) and a computer. The OPN and Bcl-2 densities were determined by Image-Pro Plus version 5.0 software (Media Cybernetics, Bethesda, MD, USA) as previously described (10). Uniform settings were used for each antibody staining. The IOD of all positive staining in each image was measured and the density of each antibody was calculated as the product of IOD/total area.

Western blot analysis. The protein levels of OPN and Bcl-2 in cell lines and tumor tissues were evaluated via western blot analysis. Total protein was extracted in lysis buffer for $45 \mathrm{~min}$ on ice. Equal amounts of protein of each sample $(50 \mu \mathrm{g})$ were denatured by heating at $100^{\circ} \mathrm{C}$ for 5 min with Laemmli loading buffer, and subsequently loaded on $10 \%$ SDS-polyacrylamide gel electrophoresis, then transferred onto polyvinylidene fluoride membranes (Millipore). Primary antibodies against OPN $(1: 1,000)$ and Bcl-2 (1:1,000) (Abcam) were used. A monoclonal antibody against GAPDH (1:1,000; Santa Cruz Biotechnology, Inc.) was used as the internal control. The bands were quantified using Gel-Pro Analyzer 4.0 (Media Cybernetics). The OPN/ GAPDH and Bcl-2/GAPDH ratios were calculated for relative quantization. Each experiment was repeated at least 3 times.

Statistical analyses. Statistical analysis was performed with SPSS 15.0 for Windows (SPSS, Chicago, IL, USA). The Kaplan-Meier method was used to calculate the survival and recurrence curves and to estimate OS and TTR. The log-rank test was used to compare TTR and OS between patients in different groups. The Spearman's rank and Fisher's exact tests were applied to demonstrate clinicopathological correlations. Univariate and multivariate analyses were performed with Cox proportional hazards model. Student's t-test was used for the comparison of data between groups; if variances within groups 


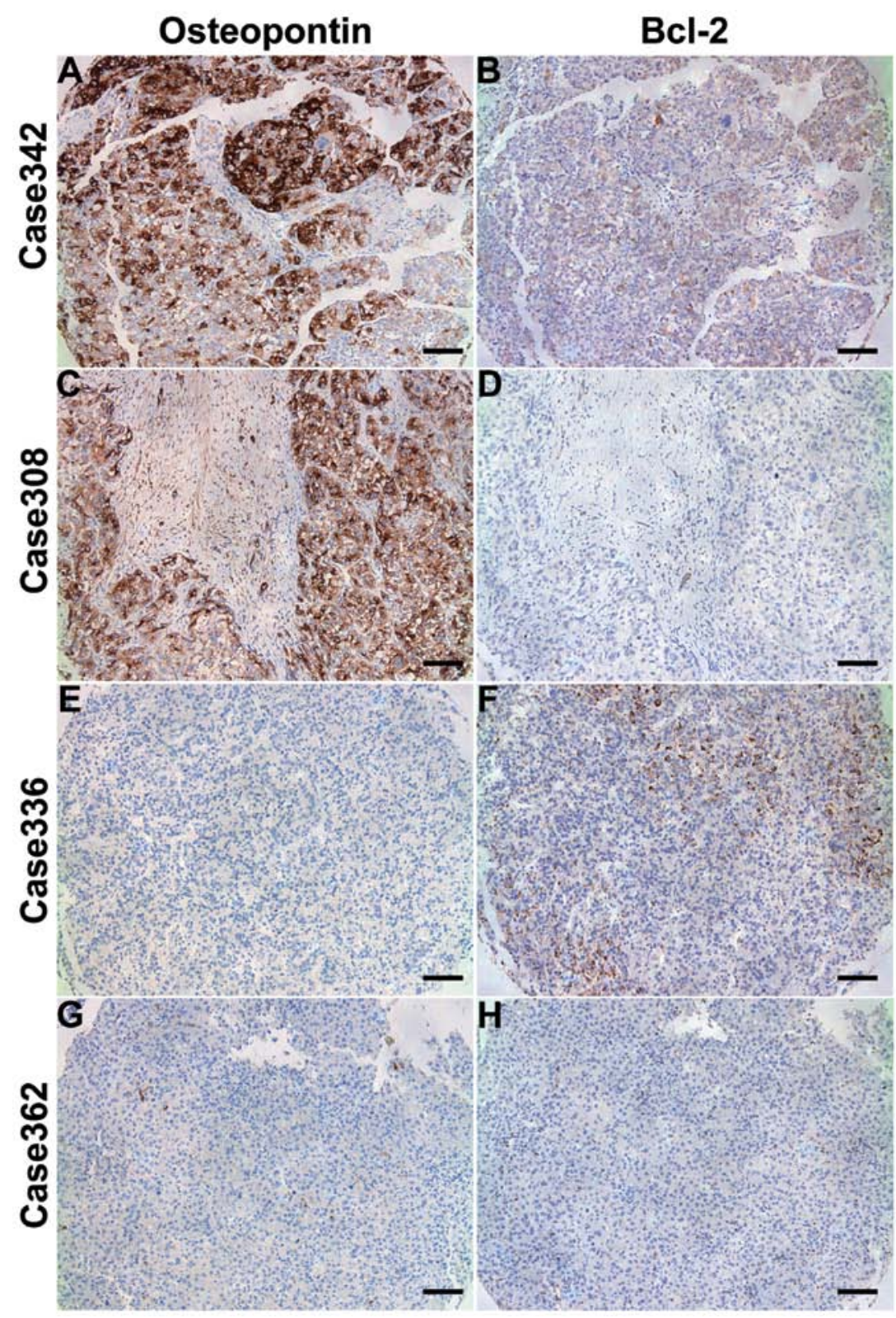

Figure 2. Different expression status of OPN and Bcl-2 detected by immunohistochemical staining in the consecutive sections of HCC tissues from the same patient. (A) HCC of case 342 is high OPN expression; (B) case 342 is high Bcl-2 expression; (C) case 308 is high OPN expression; (D) case 308 is low Bcl-2 expression; (E) case 336 is low OPN expression; (F) case 336 is high Bcl-2 expression; (G) case 336 is low OPN expression. (H) case 336 is low Bcl-2 expression. Positive cells were stained brown (magnification, $\mathrm{x} 100$ ); bar=100 $\mu \mathrm{m}$. OPN, osteopontin; HCC, hepatocellular carcinoma.

were not homogeneous, the nonparametric Mann-Whitney U test and Kruskal-Wallis $\mathrm{H}$ test were used. Values are expressed as the means \pm standard deviation. $\mathrm{p}<0.05$ was considered to indicate a statistically significant difference. For OPN and $\mathrm{Bcl}-2$ density, the cutoff for the definition of subgroups was the median value.

\section{Results}

OPN and Bcl-2 expression in HCC cell lines. After assessing OPN and Bcl-2 expression levels in MHCC97-H, MHCC97-L, HepG2 and SMMC-7721 cell lines by western blot analysis, we found that OPN expression increased with increasing metastatic potential in human $\mathrm{HCC}$ cell lines while Bcl-2 decreased with increasing metastatic potential (Fig. 1B).
To investigate the association between OPN and BCL2, we analyzed the expression level of BCL2 in HCCLM3 cells transfected with lentiviral vectors with specific miRNAs for OPN, whose OPN levels were previously analyzed (9); Bcl-2 expression increased significantly in HCCLM3 cells when OPN was knocked down (Fig. 1C).

Expression of OPN and Bcl-2 in HCC. OPN expressed highly in recurrent tumor tissue but low in non-recurrent ones by western blotting. Bcl-2 was expressed low in recurrent tumor tissue and low in non-recurrent ones. In the relative quantitative analysis below, OPN was significantly higher in recurrent tumor tissue than in non-recurrent ones ( $\left.{ }^{* *} \mathrm{p}<0.001\right)$. Bcl-2 was significantly higher in non-recurrent than in recurrent tumor tissue ( $\mathrm{p}=0.046)($ Fig. 1A). 
A

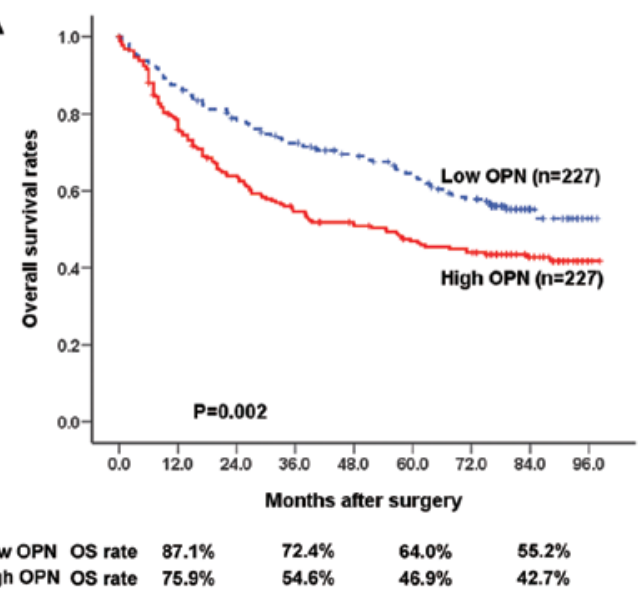

C

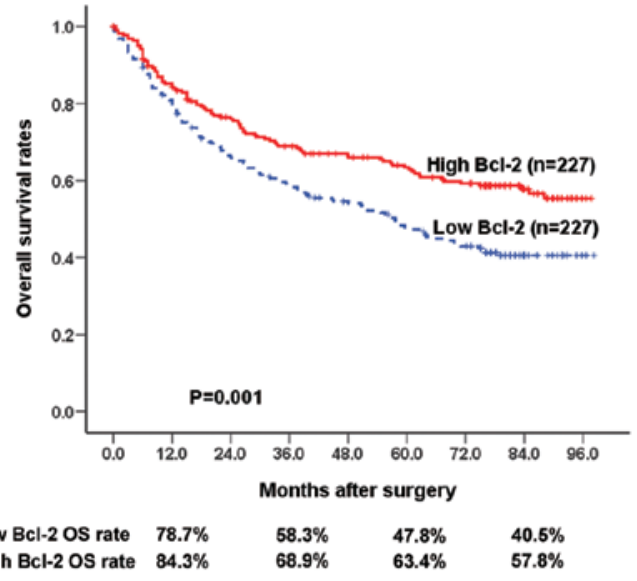

B

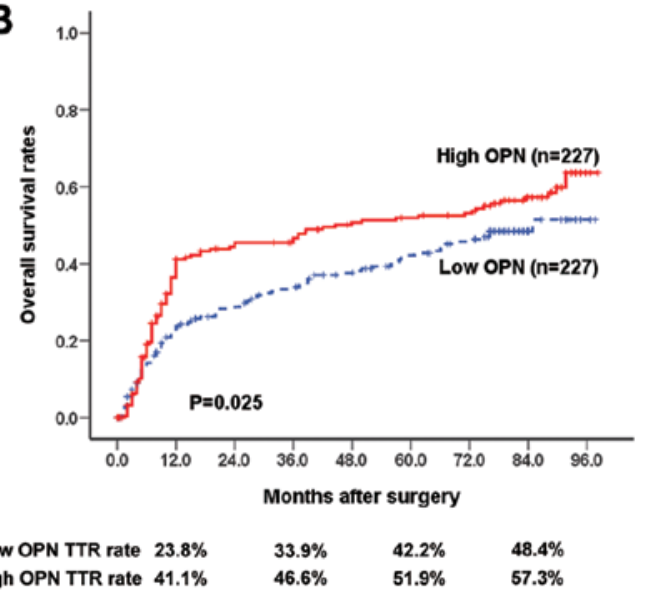

E

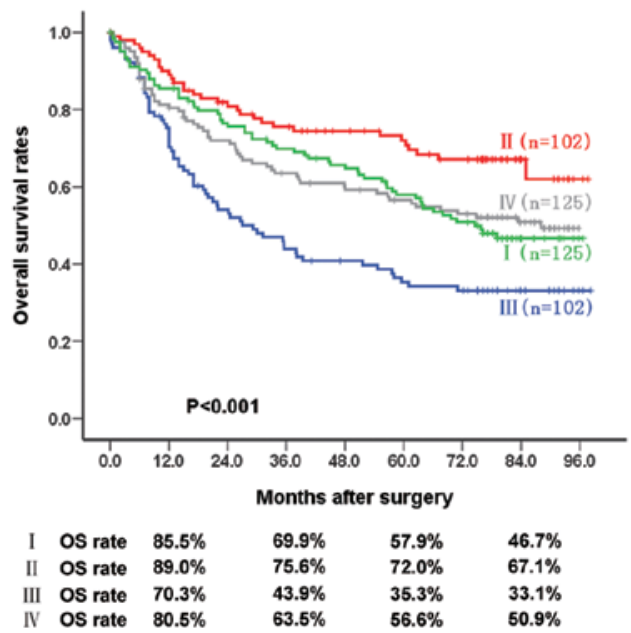

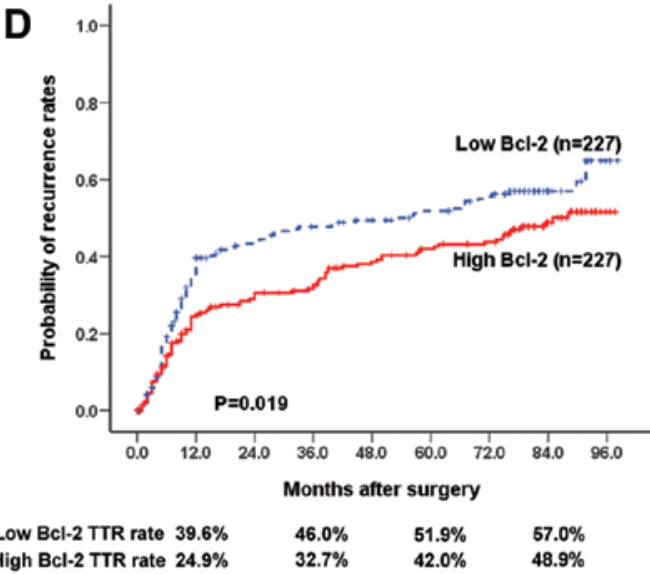

$\mathbf{F}$

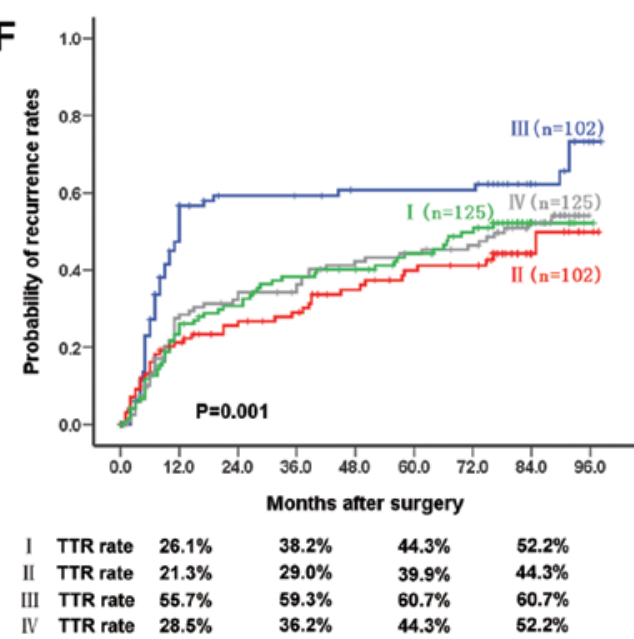

Figure 3. Prognostic significance assessed by Kaplan-Meier analysis and log-rank tests. (A and B) The 1-, 3-, 5- and 7-year overall survival rates (log-rank test, $\mathrm{p}=0.002 ; 87.1,72.4,64$ and $55.2 \%$, respectively for low OPN patients, vs. $75.9,54.6,46.9$ and $42.7 \%$, respectively for high OPN patients) and cumulative recurrence rates ( $\mathrm{p}=0.025 ; 23.8,33.9,42.2$ and $48.4 \%$, respectively for low OPN patients, vs. 41.1, 46.6, 51.9 and $57.3 \%$, respectively for high OPN patients) of low OPN patients were significantly different from those of high OPN patients. (C and D) The 1-, 3-, 5- and 7-year overall survival rates ( $\mathrm{p}=0.001 ; 78.7,58.3,47.8$ and $40.5 \%$, respectively for low Bcl-2 patients, vs. $84.3,68.9,63.4$ and $57.8 \%$, respectively for high Bcl-2 patients) and cumulative recurrence rates ( $\mathrm{p}=0.019$; 39.6, 46.0, 51.9 and 57.0\%, respectively for low Bcl-2 patients, vs. 24.9, 32.7, 42.0 and 48.9\%, respectively for high Bcl-2 patients) of low Bcl-2 patients were significantly different from those of high Bcl-2 patients. (E and F) By combining OPN and Bcl-2, the 1-, 3-, 5- and 7-year overall survival rates ( $\mathrm{p}<0.001 ; 89.0$, 75.6, 72.0 and $67.1 \%$, respectively for group II, vs. $70.3,43.9,35.3$ and $33.1 \%$, respectively for group III) and cumulative recurrence rates ( $\mathrm{p}=0.001 ; 21.3,29.0$, 39.9 and $44.3 \%$, respectively for group II, vs. 55.7, 59.3, 60.7 and $60.7 \%$, respectively for group III) of group II patients were significantly different from those of group III patients. Group I, patients with high OPN and high Bcl-2 (n=125); group II, patients with high OPN and low Bcl-2 ( $\mathrm{n}=102)$; group III, patients with low OPN and high Bcl-2 ( $\mathrm{n}=102)$; group IV, low OPN and low Bcl-2 ( $\mathrm{n}=125)$. OPN, osteopontin.

The positive staining for OPN was mainly found in $\mathrm{HCC}$ cells in the peripheral region of cancer nodules, rather than in the noncancerous hepatocytes. OPN staining was localized in the cytoplasm of HCC cells. Immunoreactivity for Bcl-2 was observed both in the cytoplasm and the nucleus of HCC cells (Fig. 2). 
Table II. Univariate analyses of factors associated with overall survival and time to recurrence.

\begin{tabular}{|c|c|c|c|c|}
\hline \multirow[b]{2}{*}{ Variable } & \multicolumn{2}{|c|}{ Overall survival } & \multicolumn{2}{|c|}{ Time to recurrence } \\
\hline & Hazard ratio $(95 \% \mathrm{CI})$ & P-value & Hazard ratio $(95 \% \mathrm{CI})$ & P-value \\
\hline Gender (male vs. female) & $0.974(0.687-1.382)$ & 0.882 & $1.066(0.735-1.545)$ & 0.737 \\
\hline Age, years (>52 vs. $\leq 52$ ) & $1.057(0.812-1.377)$ & 0.679 & $1.056(0.806-1.382)$ & 0.694 \\
\hline $\operatorname{AFP}(\mathrm{ng} / \mathrm{ml} ;>20$ vs. $\leq 20)$ & $1.597(1.196-2.131)$ & 0.001 & $1.411(1.061-1.875)$ & 0.018 \\
\hline HBsAg (positive vs. negative) & $1.766(0.999-3.122)$ & 0.050 & $2.224(1.188-4.162)$ & 0.012 \\
\hline Liver cirrhosis (yes vs. no) & $1.464(0.892-2.401)$ & 0.131 & $1.625(0.976-2.706)$ & 0.062 \\
\hline ALT (units/1; >75 vs. $\leq 75$ ) & $1.062(0.710-1.590)$ & 0.768 & $0.910(0.595-1.393)$ & 0.666 \\
\hline Tumor size $(\mathrm{cm} ;>5$ vs. $\leq 5)$ & $1.758(1.327-2.329)$ & $<0.001$ & $1.639(1.220-2.202)$ & 0.001 \\
\hline Tumor number (multiple vs. single) & $1.468(0.917-2.350)$ & 0.110 & $0.919(0.524-1.612)$ & 0.769 \\
\hline Vascular invasion (yes vs. no) & $2.041(1.560-2.671)$ & $<0.001$ & $1.590(1.197-2.112)$ & 0.001 \\
\hline BCLC stage (B/C vs. A) & $2.055(1.573-2.684)$ & $<0.001$ & $1.527(1.153-2.021)$ & 0.003 \\
\hline Tumor differentiation (III-IV vs. I-II) & $1.521(1.132-2.045)$ & 0.005 & $1.338(0.980-1.827)$ & 0.067 \\
\hline Tumor encapsulation (none vs. complete) & $1.414(1.085-1.844)$ & 0.010 & $1.268(0.968-1.660)$ & 0.084 \\
\hline OPN (high vs. low) & $1.530(1.171-1.999)$ & 0.002 & $1.359(1.036-1.782)$ & 0.027 \\
\hline Bcl-2 (high vs. low) & $0.639(0.489-0.836)$ & 0.001 & $0.727(0.554-0.953)$ & 0.021 \\
\hline \multicolumn{5}{|l|}{ Combination of OPN and Bcl-2 } \\
\hline Overall & & $<0.001$ & & 0.002 \\
\hline II vs. III & $2.726(1.784-4.164)$ & $<0.0001$ & $1.922(1.285-2.876)$ & 0.001 \\
\hline
\end{tabular}

As shown in Table I, high OPN expression in tumor tissue was significantly correlated with tumor differentiation $(\mathrm{p}<0.001)$ and HBsAg level $(\mathrm{p}=0.006)$. However, no statistically significant association was found between the Bcl-2 expression and these clinical characteristics.

Prognostic value of $\mathrm{OPN}$ and $\mathrm{Bcl}-2$ in $454 \mathrm{HCC}$ patients. Using IOD median value as the cutoff value, $454 \mathrm{HCC}$ patients were divided into 2 groups. The 1-, 3-, 5- and 7-year OS rates of high-OPN patients $(75.9,54.6,46.9$ and $42.7 \%$, respectively) were significantly lower than those of low-OPN patients $(87.1,72.4,64$ and $55.2 \%$, respectively; $\mathrm{p}=0.002$ ) (Fig. 3A). The 1-, 3-, 5- and 7-year recurrence rates of high-OPN patients $(41.1,46.6,51.9$ and $57.3 \%$, respectively) were much higher than those of low-OPN patients (23.8, 33.9, 42.2 and 48.4\%, respectively; $p=0.025)$ (Fig. 3B). The 1-, 3-, 5- and 7-year OS rates of high-Bcl-2 patients (84.3, $68.9,63.4$ and $57.8 \%$, respectively) were significantly lower than those of low-Bcl-2 patients (78.7, 58.3, 47.8 and $40.5 \%$, respectively; $\mathrm{p}=0.001$ ) (Fig. $3 \mathrm{C}$ ). The 1-, 3-, 5- and 7-year tumor recurrence rates of high $\mathrm{Bcl}-2$ patients $(24.9,32.7,42.0$ and $48.9 \%$, respectively) were much higher than those of low Bcl-2 patients $(39.6,46.0,51.9$ and $57.0 \%$, respectively; $\mathrm{p}=0.019)$ (Fig. 3D).

Combined value of OPN and Bcl-2 to predict OS or TTR. Based on the expression levels of OPN and Bcl-2, we stratified the 454 HCC patients into four groups: Group I, patients with high OPN and high Bcl-2 (n=125); Group II, high OPN and low Bcl-2 ( $\mathrm{n}=102)$; Group III, low OPN and high Bcl-2 $(n=102)$; Group IV, low OPN and low Bcl-2 $(n=125)$. The patients of Group II (high OPN/low Bcl-2) had much shorter OS and TTR compared with those of Group III (low OPN/ high Bcl-2). The 1-, 3-, 5- and 7-year OS rates of Group II $(89.0 \%, 75.6 \%, 72.0 \%$ and $67.1 \%$, respectively) were significantly lower compared with Group III (70.3\%, 43.9\%, 35.3\% and $33.1 \%$, respectively; $\mathrm{p}<0.001$ ) (Fig. 3E). The 1-, 3-, 5- and 7-year recurrence rates of Group II $(21.3 \%, 29.0 \%, 39.9 \%$ and $44.3 \%$, respectively) were significantly lower than those of patients in Group III $(55.7 \%, 59.3 \%, 60.7 \%$ and $60.7 \%$, respectively; $\mathrm{p}=0.001$ ) (Fig. 3F).

Univariate and multivariate analyses of the prognostic values of OPN and Bcl-2 in HCC patients. To further evaluate the prognostic value of OPN and Bcl-2 for HCC patients, univariate and multivariate analyses were performed with the clinicopathological characteristics as well as the OPN and Bcl-2 levels (Tables II and III). In the univariate analysis, serum AFP level, tumor size, tumor number, vascular invasion, BCLC stage, tumor differentiation and tumor encapsulation were shown to be associated with OS of HCC patients, and serum AFP, tumor size, vascular invasion, BCLC stage with TTR. The OPN and Bcl-2 expression levels in HCC tissues were also significantly associated with both OS and TTR.

Individual characteristics that showed significance by univariate analysis were adopted as covariates in a multivariate Cox proportional hazards model, and then combined 
Table III. Multivariate analyses of factors associated with overall survival and time to recurrence.

\begin{tabular}{|c|c|c|c|c|}
\hline \multirow[b]{2}{*}{ Variable } & \multicolumn{2}{|c|}{ Overall survival } & \multicolumn{2}{|c|}{ Time to recurrence } \\
\hline & Hazard ratio $(95 \% \mathrm{CI})$ & P-value & Hazard ratio $(95 \% \mathrm{CI})$ & P-value \\
\hline $\operatorname{AFP}(\mathrm{ng} / \mathrm{ml} ;>20$ vs. $\leq 20)$ & $1.397(1.038-1.880)$ & 0.027 & $1.263(0.941-1.694)$ & NS \\
\hline Tumor size $(\mathrm{cm} ;>5$ vs. $\leq 5)$ & $1.826(1.364-2.444)$ & $<0.001$ & $1.697(1.253-2.299)$ & 0.001 \\
\hline HBsAg (positive vs. negative ) & $1.373(0.767-2.458)$ & NS & & \\
\hline Vascular invasion (yes vs. no ) & $1.648(1.246-2.181)$ & $<0.001$ & $1.381(1.029-1.855)$ & 0.032 \\
\hline Tumor encapsulation (none vs. complete) & $1.421(1.084-1.863)$ & 0.011 & & \\
\hline Tumor differentiation (III-IV vs. I-II) & $1.187(0.873-1.615)$ & NS & & \\
\hline OPN (high vs. low) & $1.652(1.250-2.183)$ & $<0.001$ & $1.478(1.115-1.959)$ & 0.007 \\
\hline Bcl-2 (high vs. low) & $0.532(0.404-0.701)$ & $<0.001$ & $0.632(0.478-0.835)$ & 0.001 \\
\hline \multicolumn{5}{|l|}{ Combination of OPN and Bcl-2 } \\
\hline Overall & & $<0.001$ & & 0.001 \\
\hline II vs. III & $3.725(2.330-5.954)$ & $<0.0001$ & $2.244(1.469-3.429)$ & 0.0002 \\
\hline
\end{tabular}

Multivariate analysis, Cox proportional hazards regression model. 95\% CI, 95\% confidence interval; AFP, $\alpha$-fetoprotein; NS, not significant (Cox proportional hazards regression model); BCLC, Barcelona Clinic Liver Cancer staging system. I, OPN high and Bcl-2 high; II, OPN high and Bcl-2 low; III, OPN low and Bcl-2 high; IV, OPN low and Bcl-2 low. HBsAg, hepatitis B surface antigen; OPN, osteopontin.

A

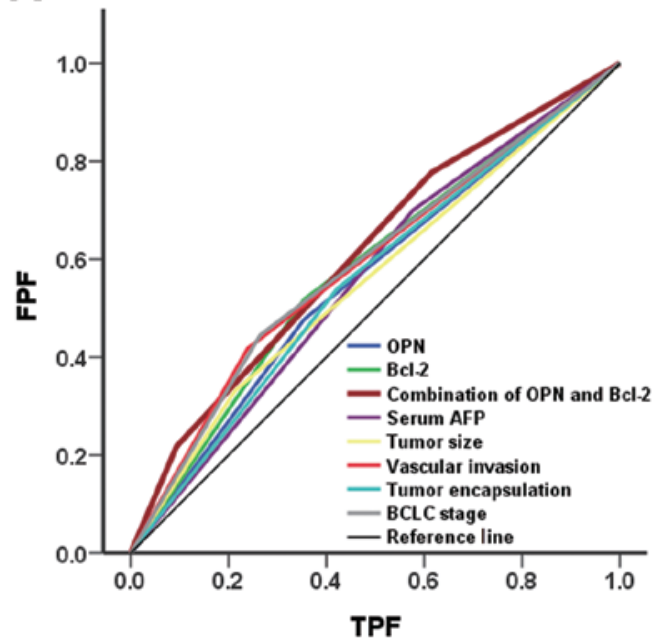

C

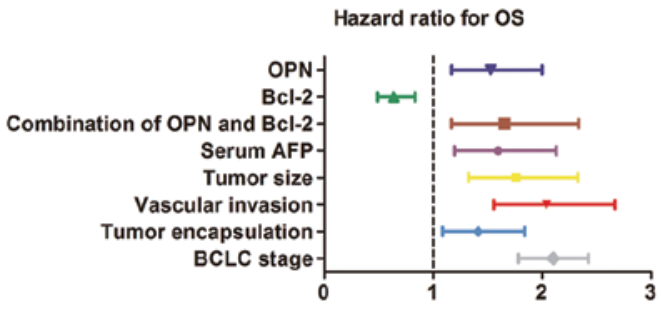

B

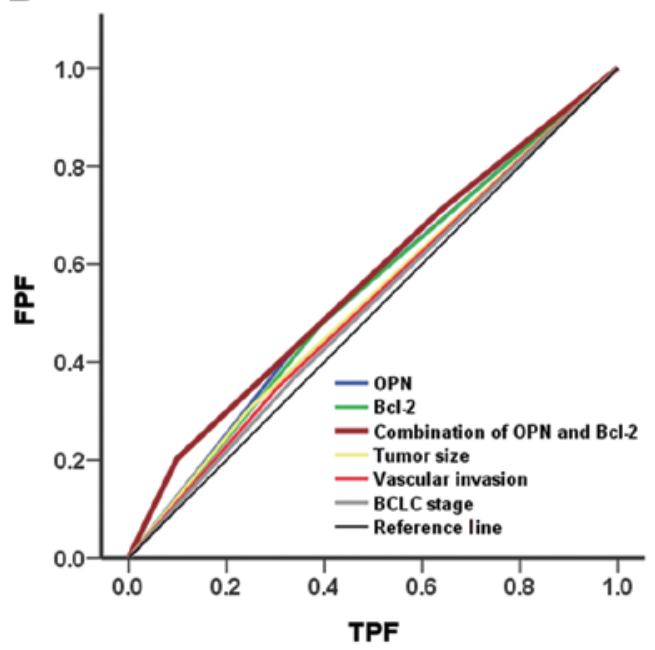

D

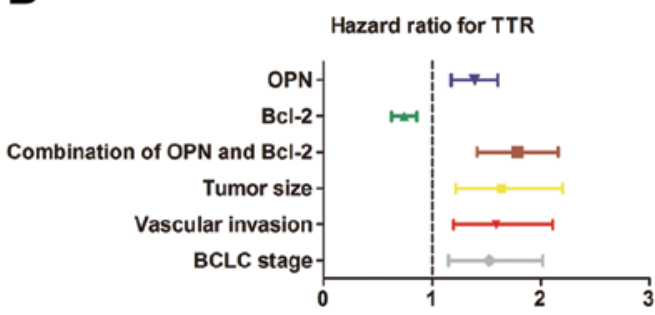

Figure 4. (A and B) ROC analysis was performed. For both mortality and recurrence, the predictive value of the combination was the best. FPF, false-positive fraction; TPF, true-positive fraction. (C and D) Clinicopathological factors showing significance by univariate survival analysis and the combination of OPN and Bcl-2 were adopted for hazard ratio analysis. As shown in the figure, Bcl-2 was a protective factor in HCC prognosis. OPN, osteopontin; HCC, hepatocellular carcinoma.

variables were further analyzed. Both OPN and Bcl-2 were demonstrated to be independent prognostic indicators for OS $(\mathrm{p}<0.001$ both for OPN and Bcl-2) and TTR $(\mathrm{p}=0.004$ for OPN, $\mathrm{p}=0.002$ for Bcl-2). Moreover, combination of OPN with Bcl-2 was an even more powerful independent prognostic indicator for both OS $(\mathrm{p}<0.001)$ and TTR $(\mathrm{p}<0.001)$. Hazard ratio analysis showed $\mathrm{Bcl}-2$ was a protective factor in $\mathrm{HCC}$ prognosis (Fig. 4C and D). 
ROC analysis for OPN and Bcl-2. Clinicopathological factors showing significance by multivariate survival analysis and the combination of OPN and Bcl-2 were adopted for ROC analysis. For both mortality and recurrence, the predictive value of the combination of OPN and Bcl-2 was the best. The area under the curve of this combination was 0.622 (95\% CI, 0.570-0.673; $\mathrm{p}<0.001)$ for mortality and $0.569(95 \% \mathrm{CI}, 0.516-0.622$; $\mathrm{p}=0.011$ ) for recurrence (Fig. 4A and B).

\section{Discussion}

The poor prognosis of $\mathrm{HCC}$ is often the result of late diagnosis or high rate of recurrence after surgery. Metastasis is a major complication of HCC pathogenesis that typifies a poor prognosis. Current approaches are hindered by a lack of clinically useful biomarkers. OPN has been implicated as an important mediator of tumor metastasis and has been investigated for use as a biomarker for advanced disease and as a potential therapeutic target in the regulation of cancer metastasis. It is a secreted multifunctional glycoprotein expressed at high levels in tumors and the surrounding stroma of numerous types of cancer, including liver (15-17). Increased serum and plasma OPN levels are associated with advanced-stage lung, breast (18), colon and prostate carcinomas $(19,20)$. OPN expression can predict high-grade, late-stage and early-recurrence HCC (21).

Our previous study identified OPN as a predictive marker for HCC and a molecular target of the HCC metastatic phenotype. The data demonstrated a correlation between OPN mRNA expression and primary HCC metastasis (22). Furthermore, increased OPN levels were associated with increased aggressiveness and metastatic potential of HCC and positively correlated with poor prognosis and early tumor recurrence in HCC patients $(23,24)$.

In vitro, we found that OPN expression increased with increasing metastatic potential in human HCC cell lines while Bcl-2 decreased with increasing metastatic potential. These data support the findings in HCC TMA and demonstrated that OPN may interact with Bcl-2 in HCC tumors and cells. Thus, we performed miRNAs for OPN in MHCC97-H cells which have a high expression level of OPN. We found that OPN decreased in shOPN MHCC97-H cells significantly compared with mock and shNon ones. Bcl-2 expression increased significantly in shOPN MHCC97-H cells. This discovery appears to be in contrast to previous reports. For example, in malignant gliomas, OPN siRNA induced clear upregulation of Bax expression, downregulation of Bcl-2, Bcl-xL (25). Our findings are based on large cohort of HCC patients. Hung et al (8) reported that HBV pre-S2D increased Bcl-2 expression, indicating that the cohort of HCC patients, who had $92.1 \%$ HBV infection, will have some difference compared with other types of tumors. Furthermore, patients with higher OPN levels had significantly shorter median survival time and recurrence time than the lower ones. It was confirmed that OPN levels are also significantly higher in recurrent tumor tissues than in the non-recurrent ones by western blotting $(\mathrm{p}<0.001)$. In previous reports, an elevated plasma level of OPN was regarded as a potential prognostic biomarker, and overexpression of OPN was closely correlated with intrahepatic metastasis, early recurrence, and a poorer prognosis; this result is similar to ours in tumor tissue $(21,22,24,26)$.
Improving OPN predicting efficiency for HCC patients needs to be elucidated. It is necessary to combine OPN and Bcl-2 to deal with this issue. Therefore, we used TMA contained $454 \mathrm{HCC}$ cases to examine both OPN and Bcl-2 expression levels. Inversely, the higher BCL-2 levels were associated with longer median survival time and recurrence time in HCC patients. The co-index of OPN/Bcl-2 was an independent prognostic factor for both overall survival $(p<0.001)$ and time to recurrence $(\mathrm{p}=0.001)$.

Bcl-2 overexpression has since been reported in several tumor types and is often correlated with poor survival (27). Bcl-2 is best known for its ability to suppress apoptosis and belongs to a group of related proteins that are key regulators of apoptosis or programmed cell death (28). The phenomenon of Bcl-2 expression in our study seems to deviate from the common notion that genes are 'vicious' factors. Furthermore, numerous studies have suggested that the gene may undergo conversion from protector to killer under some circumstances. Dawson et al (7) performed a study on breast cancer in 11,212 women and found that increasing expression levels of Bcl-2 predict better survival in early breast cancer and Bcl-2 is a suitable time-independent prognostic marker in early breast cancer.

Several possible mechanisms have been founf regarding this seemingly contradictory phenomenon. First, mutations in translocated Bcl-2 alleles have been identified in lymphomas which ablate the aspartic acid residue required for caspase cleavage (29). Of note, proteolytic removal of N-terminal sequences by caspase-mediated cleavage could reverse the phenotype of Bcl-2 (30). Second, the orphan nuclear receptor Nur77 can be induced to translocate from nucleus to cytosol, binding Bcl-2, and inducing a conformational change in Bcl-2 that probably mimics what happens during caspase cleavage, exposing the normally buried $\mathrm{BH} 3$ domain of $\mathrm{Bcl}-2$ and causing it to function as a pro-apoptotic protein (31). Another hypothesis (28) considers increased expression of Bcl-2 protein may also disrupt the balance with other members of the Bcl-2 family, including the expression of pro-apoptotic proteins. The molecular nature of such pores and how anti-apoptotic Bcl-2 family proteins may regulate them remains to be clarified. A potentially similar mechanism identified for Bcl-xL showed that lipid modifications of K-Ras can promote its association with Bcl-xL in mitochondria and induce apoptosis (32). Thus, depending on which proteins Bcl-2 and Bcl-xL interact with, their phenotypes can be converted from anti- to pro-apoptotic (33), providing a potential explanation for why high levels of Bcl-2 expression are sometimes associated with improved patient prognosis (34).

Most studies have demonstrated that HCC tissues do not express or have only a low positive rate of $\mathrm{Bcl}-2$ protein. Sometimes the positive rate of Bcl-2 in HCC tissues was lower than that in the non-tumor liver tissues immediately adjacent to HCC tissues (35). The mechanism of this phenomenon remains unclear. There may be specific characteristics of the regulation of Bcl-2 in HCC.

In conclusion, we showed that OPN/Bcl-2 expression is a promising independent predictor of recurrence and survival in HCC that may aid in the therapy of HCC patients. Bcl-2 levels may be regulated by OPN in the HCC microenvironment. The HBV background of the patients may have some unique influence on Bcl-2 expression (8). Related mechanisms should be investigated in future studies. 


\section{References}

1. Wai PY and Kuo PC: Osteopontin: regulation in tumor metastasis. Cancer Metastasis Rev 27: 103-118, 2008.

2. Khan SA, Lopez-Chua CA, Zhang J, Fisher LW, Sørensen ES and Denhardt DT: Soluble osteopontin inhibits apoptosis of adherent endothelial cells deprived of growth factors. J Cell Biochem 85: 728-736, 2002.

3. Frenzel A, Grespi F, Chmelewskij W and Villunger A: Bcl2 family proteins in carcinogenesis and the treatment of cancer. Apoptosis 14: 584-596, 2009.

4. Yoo SH, Yoon YG, Lee JS, et al: Etoposide induces a mixed type of programmed cell death and overcomes the resistance conferred by Bcl-2 in Hep3B hepatoma cells. Int J Oncol 41: 1443-1454, 2012.

5. Spampanato C, De Maria S, Sarnataro M, et al: Simvastatin inhibits cancer cell growth by inducing apoptosis correlated to activation of Bax and downregulation of BCL-2 gene expression. Int J Oncol 40: 935-941, 2012.

6. Callagy GM, Pharoah PD, Pinder SE, et al: $\mathrm{Bcl}-2$ is a prognostic marker in breast cancer independently of the Nottingham Prognostic Index. Clin Cancer Res 12: 2468-2475, 2006.

7. Dawson SJ, Makretsov N, Blows FM, et al: BCL2 in breast cancer: a favourable prognostic marker across molecular subtypes and independent of adjuvant therapy received. Br J Cancer 103: $668-675,2010$

8. Hung JH, Teng YN, Wang LH, et al: Induction of Bcl-2 expression by hepatitis B virus pre-S2 mutant large surface protein resistance to 5-fluorouracil treatment in Huh-7 cells. PLoS One 6: e28977, 2011.

9. Zhao J, Dong L, Lu B, et al: Down-regulation of osteopontin suppresses growth and metastasis of hepatocellular carcinoma via induction of apoptosis. Gastroenterology 135: 956-968, 2008

10. Zhu XD, Zhang JB, Zhuang PY, et al: High expression of macrophage colony-stimulating factor in peritumoral liver tissue is associated with poor survival after curative resection of hepatocellular carcinoma. J Clin Oncol 26: 2707-2716, 2008.

11. Llovet JM, Di Bisceglie AM, Bruix J, et al: Design and endpoints of clinical trials in hepatocellular carcinoma. J Natl Cancer Inst 100: 698-711, 2008.

12. Huang H, Zhang XF, Zhou HJ, et al: Expression and prognostic significance of osteopontin and caspase-3 in hepatocellular carcinoma patients after curative resection. Cancer Sci 101: 1314-1319, 2010.

13. Gao Q, Qiu SJ, Fan J, et al: Intratumoral balance of regulatory and cytotoxic $\mathrm{T}$ cells is associated with prognosis of hepatocellular carcinoma after resection. J Clin Oncol 25: 2586-2593, 2007.

14. Ding ZB, Shi YH, Zhou J, et al: Association of autophagy defect with a malignant phenotype and poor prognosis of hepatocellular carcinoma. Cancer Res 68: 9167-9175, 2008.

15. Butler WT and Ritchie $\mathrm{H}$ : The nature and functional significance of dentin extracellular matrix proteins. Int J Dev Biol 39: 169-179, 1995.

16. Coppola D, Szabo M, Boulware D, et al: Correlation of osteopontin protein expression and pathological stage across a wide variety of tumor histologies. Clin Cancer Res 10: 184-190, 2004.

17. Rittling SR and Chambers AF: Role of osteopontin in tumour progression. Br J Cancer 90: 1877-1881, 2004.

18. Singhal H, Bautista DS, Tonkin KS, et al: Elevated plasma osteopontin in metastatic breast cancer associated with increased tumor burden and decreased survival. Clin Cancer Res 3: 605-611, 1997.
19. Fedarko NS, Jain A, Karadag A, Van Eman MR and Fisher LW: Elevated serum bone sialoprotein and osteopontin in colon, breast, prostate, and lung cancer. Clin Cancer Res 7: 4060-4066, 2001.

20. Hotte SJ, Winquist EW, Stitt L, Wilson SM and Chambers AF: Plasma osteopontin: associations with survival and metastasis to bone in men with hormone-refractory prostate carcinoma. Cancer 95: 506-512, 2002.

21. Pan HW, Ou YH, Peng SY, et al: Overexpression of osteopontin is associated with intrahepatic metastasis, early recurrence, and poorer prognosis of surgically resected hepatocellular carcinoma. Cancer 98: 119-127, 2003.

22. Ye QH, Qin LX, Forgues M, et al: Predicting hepatitis B virus-positive metastatic hepatocellular carcinomas using gene expression profiling and supervised machine learning. Nat Med 9: 416-423, 2003.

23. Sun J, Xu HM, Zhou HJ, et al: The prognostic significance of preoperative plasma levels of osteopontin in patients with TNM stage-I of hepatocellular carcinoma. J Cancer Res Clin Oncol 136: 1-7, 2010.

24. Zhang $\mathrm{H}$, Ye QH, Ren $\mathrm{N}$, et al: The prognostic significance of preoperative plasma levels of osteopontin in patients with hepatocellular carcinoma. J Cancer Res Clin Oncol 132: 709-717, 2006.

25. Yan W, Qian C, Zhao P, et al: Expression pattern of osteopontin splice variants and its functions on cell apoptosis and invasion in glioma cells. Neuro Oncol 12: 765-775, 2010.

26. Xie H, Song J, Du R, et al: Prognostic significance of osteopontin in hepatitis B virus-related hepatocellular carcinoma. Dig Liver Dis 39: 167-172, 2007.

27. Fesik SW: Promoting apoptosis as a strategy for cancer drug discovery. Nat Rev Cancer 5: 876-885, 2005.

28. Cory S, Huang DC and Adams JM: The Bcl-2 family: roles in cell survival and oncogenesis. Oncogene 22: 8590-8607, 2003.

29. Tanaka S, Louie D, Kant J and Reed JC: Application of a PCR-mismatch technique to the BCL-2 gene: detection of point mutations in BCL-2 genes of malignancies with A $t(14,18)$. Leukemia 6 (Suppl 3): S15-S19, 1992.

30. Cheng EH, Kirsch DG, Clem RJ, et al: Conversion of Bcl-2 to a Bax-like death effector by caspases. Science 278: 1966-1968, 1997.

31. Lin YP, Zhu BZ, Yang MC, et al: Bcl-2 overexpression inhibits tetrachlorohydroquinone-induced apoptosis in NIH3T3 cells: a possible mechanism for tumor promotion. Mol Carcinog 40: 24-33, 2004.

32. Bivona TG, Quatela SE, Bodemann BO, et al: $\mathrm{PKC}$ regulates a farnesyl-electrostatic switch on K-Ras that promotes its association with Bcl-XL on mitochondria and induces apoptosis. Mol Cell 21: 481-493, 2006.

33. Schwartz PS and Hockenbery DM: Targeted therapies for epithelial cancers: in vivo efficacy of the BCL-2/BCL-XL inhibitor 2-MeAA. Cancer Biol Ther 6: 465-466, 2007.

34. Silvestrini R, Veneroni S, Daidone MG, et al: The Bcl-2 protein: a prognostic indicator strongly related to $\mathrm{p} 53$ protein in lymph node-negative breast cancer patients. J Natl Cancer Inst 86: 499-504, 1994

35. Tsamandas AC, Thomopoulos K, Gogos C, et al: Expresssion of bcl-2 oprotein in cases of acute and chronic viral hepatitis type B and type C: a clinicopathologic study. Dig Dis Sci 47: 1618-1624, 2002 . 